\title{
Difficulties with Re-collapsing Models in Closed Isotropic Loop Quantum Cosmology
}

\author{
Daniel Green and William G. Unruh \\ Department of Physics and Astronomy \\ University of British Columbia \\ Vancouver, BC, Canada, V6T $1 Z 1$ \\ email: drgreen@stanford.edu, unruh@physics.ubc.ca
}

\begin{abstract}
The use of techniques from loop quantum gravity for cosmological models may solve some difficult problems in quantum cosmology. The solutions under a number of circumstances have been well studied. We will analyse the behaviour of solutions in the closed model, focusing on the behaviour of a universe containing a massless scalar field. The asymptotic behaviour of the solutions is examined, and is used to determine requirements of the initial conditions.
\end{abstract}

\section{INTRODUCTION}

On large scales, the description of the universe provided by general relativity is consistent with all measurements to date. The cosmological models that have been generated seem to account for much of the history of the universe. However, the cosmological singularity that appears in this approach has suggested that a quantum theory of cosmology is required. Most studies of quantum cosmology have involved mini-superspace models. Homogeneity (and sometimes isotropy) is used to limit the degrees of freedom of the classical framework to a finite number. The reduced phase space is then quantized. Unfortunately, most such quantizations have been unable to provide a consistent solution to the singularity and initial value problems. Furthermore, the absence of time has lead to additional interpretation problems.

In hopes of resolving these issues, the tools of loop quantum gravity have been applied to cosmology in a similar way. Loop quantum cosmology (LQC) involves the same reduction of the phase space as other mini-superspace models; however, the system is quantized using the same techniques as the full theory of loop quantum gravity. In other mini-superspace models, the quantization is not necessarily related to that of a quantum theory of gravity. This is thought to be responsible for many of the difficulties that arise.

The results claimed by loop quantum cosmology to date have been impressive. First of all, it has been argued that the cosmological singularity is naturally removed in this framework [1]. Secondly, the initial conditions can be imposed from the behaviour of the equation itself [2]. Finally, classically divergent quantities are bounded in this framework(e.g. [7]). This introduces modifications to the evolution of the universe that seem to produce inflation [3] and eliminate chaos in the Bianchi IX model [4].

However, much of the successes of LCQ have come from the semi-classical analysis of the results. The interpretation of the quantum states themselves is not entirely clear. In particular, as with the Wheeler-DeWitt equation, 'time' does not appear as an explicit variable in the quantum framework. In the flat model, one typically picks the scale factor to represent a 'time' variable. From this, one can consider the evolution of the other degrees of freedom with respect to that time. However, in closed models where the universe recollapses classically, this interpretation seems less intuitive. In particular, the same volume corresponds, in general, to two different times. Furthermore, the classical solution attains a maximum volume, which one would expect to apply in the quantum case as well. Therefore, the probability should decay for large volumes.

Much of the work to date in LQC has involved flat models. This is not unreasonable since measurements indicate that we will in a very nearly flat universe. However, if one wants to address current cosmological models, one must be capable of addressing the 'flatness problem' associated with big bang cosmologies. As such, one must be able to understand the flat model as a limit of the closed model.

Because of the many issues involving closed cosmological models, it seems necessary to understand the nature of these solutions in loop quantum cosmology. In this paper, we will examine the solutions of the closed model in loop quantum cosmology in the presence of a scalar field. In particular, we will focus on the asymptotic behaviour of the model and how one imposes initial conditions in the exact solutions. Our analysis suggests that one cannot impose initial conditions that produce bounded solutions without introducing additional problems.

\section{THE CLOSED ISOTROPIC MODEL}

Loop quantum gravity is constructed by quantizing the phase space generated by the three-space connection variable $A$ and its conjugate $E$. In the isotropic model, these variables can be written in terms of the symmetry generators of 
the space and real multipliers. The end result is a phase space defined by the real variables $c$ and $p[7]$ such that

$$
\{c, p\}=\frac{1}{3} \kappa \gamma .
$$

where $\kappa=8 \pi G$ and $\gamma$ is the Barbero-Immirzi parameter. $c$ represents the degrees of freedom associated with the connection $A$ and is given by,

$$
c=\Gamma-\gamma K,
$$

where $\Gamma$ is the spin connection and $K$ is the extrinsic curvature. The spin connection is related to the intrinsic curvature of the spacial hypersurface, such that $\Gamma=0, \frac{1}{2}$ in the flat and closed models respectively. The conjugate momenta, $p$, is related to the scale factor $a$ through $|p|=a^{2}$. With the Hamiltonian constraint,

$$
H=-\frac{6}{\gamma^{2} \kappa} \operatorname{sgn}(p) \sqrt{|p|}\left(c^{2}-c+\frac{1+\gamma^{2}}{4}\right)=0
$$

this classical phase space describes isotropic cosmologies in general relativity.

When quantizing the phase space of the full theory, one uses smeared variables rather than the basic variables themselves [5]. Since one would like to follow the full theory as much as possible, the holonomies of the reduced phase space can be defined in a similar way. In this case, the integration can be taken over straight lines because of the symmetries, giving the holonomies of $A[7]$ as

$$
h_{e}=P \exp \left(\int_{e} A\right)=\cos (c \mu)+2 \sin (c \mu)\left(\dot{e}^{a} \omega_{a}^{i}\right) \tau^{i}
$$

where $\mu \in(-\infty, \infty)$ gives the length of the integration (which is not a physical length) and $P$ denotes path ordering. This leads to an orthonormal basis of states defined by

$$
|\mu\rangle=\exp \left(\frac{i \mu c}{2}\right)
$$

From here, one defines $\hat{p}=-i \frac{l_{p}^{2} \gamma}{3} \frac{d}{d c}$ which is an eigenfunction of these states with eigenvalue $p_{\mu} \equiv \frac{\mu \gamma l_{p}^{2}}{6}$. From the classical relation $V=|p|^{3 / 2}$, one can define

$$
\hat{V}|\mu\rangle=\left(\frac{|\mu| \gamma l_{p}^{2}}{6}\right)^{3 / 2}|\mu\rangle \equiv V_{\mu}|\mu\rangle
$$

This is a well-defined operator since $\hat{p}$ is diagonal. In fact, this can be exploited to produce an operator for any positive power of $p$. From these simple operators and the holonomies, one can produce operator representations of more complicated quantities. The action of holonomies on the basis states can be determined by expanding in imaginary exponentials and using the relation

$$
e^{i \frac{c}{2}}|\mu\rangle=|\mu+1\rangle
$$

Using the above relations, one can define an inverse volume operator

$$
\widehat{a^{-3}}|\mu\rangle=\left(\frac{9}{j(j+1)(2 j+1) \gamma l_{p}^{2} l} \sum_{k=-j}^{j} k p_{\mu+2 k}^{l}\right)^{\frac{3}{2-2 l}}|\mu\rangle \equiv d_{j, l}(\mu)|\mu\rangle,
$$

where $j$ and $l$ parameterize the ambiguities involved in defining the $V^{-1}$ operator from classical expressions [11,12], where $l \in(0,1)$ and $j$ is a positive integer. The integer $j$ arises from our freedom to use different representations of $\mathrm{SU}(2)$. The parameter $l$ arises from the fact that classically $V^{-1}=\left(V^{l-1}\right)^{\frac{1}{1-l}}$. However, the operators for $V^{l-1}$ will not preserve this identity on all scales. Asymptotically, this expression will behave like $V_{\mu}^{-1}$, for all allowed values of $l$ and $j$, since

$$
p_{\mu+m}^{l}-p_{\mu-m}^{l}=\left(\frac{\mu \gamma l_{p}^{2}}{6}\right)^{l}\left[\left(1+\frac{m}{\mu}\right)^{l}-\left(1-\frac{m}{\mu}\right)^{l}\right] \approx\left(\frac{\gamma l_{p}^{2}}{6}\right)^{l}(2 l m) \mu^{l-1}
$$


when $\mu \gg m$. However, on very small scales, the inverse volume operator is bounded where the classical value is not. The precise behaviour of the eigenvalues on these small scales depends critically on $j$ and $l$.

In order to determine the physical states and observables we need to define a Hamiltonian constraint operator. One can try to construct the operator along the lines of the full theory [5] where the Euclidian and Lorentzian constraints are quantized separately. This procedure was performed in [9] for both the flat and closed models. For the flat model, this approach works well; however, in the closed model, the quantizations is unstable [10]. Therefore another approach was found to be necessary for the closed model.

There is a different approach is based on the techniques used for the Bianchi IX model $[14,15]$. Since $\Gamma=\frac{1}{2}$ for the closed model, one can produce an operator for the Hamiltonian constraint using $c-\Gamma$ in place of $c$. This method is likely to have less in common with the full theory since it exploits the form of $\Gamma$, but we can use the same techniques as the flat case. The resulting Hamiltonian constraint operator was found to be [8]

$\hat{C}_{\text {grav }}=96 i\left(\gamma^{3} \mu_{o}^{3} l_{p}^{2}\right)^{-1}\left(\sin ^{2}\left(\mu_{o} \frac{(c-\Gamma)}{2}\right) \cos ^{2}\left(\mu_{o} \frac{(c-\Gamma)}{2}\right)-\frac{\gamma^{2} \mu_{o}^{2}}{4}\left(\Gamma^{2}-\Gamma\right)\right)\left(\sin \left(\mu_{o} \frac{c}{2}\right) \hat{V} \cos \left(\mu_{o} \frac{c}{2}\right)-\cos \left(\mu_{o} \frac{c}{2}\right) \hat{V} \sin \left(\mu_{o} \frac{c}{2}\right)\right)$

where the sines and cosines have the same action on states as previously described. $\mu_{o} \in(0, \infty)$ is an ambiguity parameter related to the 'length' of holonomies used to construct the constraint. The $\Gamma$ terms in the trigonometric operators will be pulled out to form a complex exponential multiplier in the eigenvalue. As in the full theory, any matter Hamiltonian can be added directed to this operator to form a full Hamiltonian constraint.

From the constraint operator proposed in (10), one can define the physical states of the system; ie, those that are annihilated by the constraint operator. The obvious starting point is the volume eigenfunctions. The action of the constraint operator on a volume state is

$$
\hat{C}_{\text {grav }}|\mu\rangle=3\left(\gamma^{3} \kappa l_{p}^{2}\right)^{-1}\left(V_{\mu+\mu_{o}}-V_{\mu-\mu_{o}}\right)\left(e^{2 i \Gamma \mu_{o}}\left|\mu+4 \mu_{o}\right\rangle-\left(2-4 \gamma^{2} \mu_{o}^{2}\left(\Gamma^{2}-\Gamma\right)\right)|\mu\rangle+e^{-2 i \Gamma \mu_{o}}\left|\mu-4 \mu_{o}\right\rangle\right) .
$$

Clearly the volume eigenstates, other than the zero volume state, are not annihilated. Therefore, we will write any physical state as a sum over volume states via

$$
|\Psi\rangle=\sum_{\mu} s_{\mu}(\phi)|\mu\rangle
$$

where $\phi$ denotes the matter dependence. Therefore, imposing $\hat{C}|\Psi\rangle=0$ yields the condition

$$
\begin{array}{r}
\left(V_{\mu+5 \mu_{o}}-V_{\mu+3 \mu_{o}}\right) e^{-i 2 \Gamma \mu_{o}} s_{\mu+4 \mu_{o}}-\left(2-4 \mu_{o}^{2} \gamma^{2}\left(\Gamma^{2}-\Gamma\right)\right)\left(V_{\mu+\mu_{o}}-V_{\mu-\mu_{o}}\right) s_{\mu}+\left(V_{\mu-3 \mu_{o}}-V_{\mu-5 \mu_{o}}\right) e^{i 2 \Gamma \mu_{o}} s_{\mu-4 \mu_{o}} \\
=-\frac{1}{3} \mu_{o}^{3} \gamma^{3} \kappa l_{p}^{2} \hat{H}_{\text {matt }} s_{\mu},
\end{array}
$$

where the matter Hamiltonian, $H_{\text {matt }}$, has been added directly to the constraint, as in the full theory [6]. This is the discrete equation that describes the state of the universe. Therefore, given a matter Hamiltonian, we use (13) to determine the coefficients of the physical state. However, one should keep in mind that the universe is not in one volume state that 'evolves' according to this equation. The states are only those that include the sum of all volume states. In the flat model, the parameter $\mu$ is frequently used to represent 'time'. However, it is not clear that this interpretation applies in general.

\section{EXACT SOLUTIONS}

In order to find exact solutions, we will need to choose a form of matter. For simplicity, we will use a scalar field with the Hamiltonian

$$
\hat{H}_{\text {matt }}=\frac{1}{2} \hat{a}^{-3} p_{\phi}^{2}=-\frac{\hbar^{2}}{2} a^{-3} \frac{d^{2}}{d \phi^{2}},
$$

where $\hat{a}^{-3}$ is defined as in (8). We assume that the coefficients have the form

$$
s_{\mu}(\phi)=c_{\mu} e^{i \tilde{\omega} \phi},
$$

where $\tilde{\omega}$ is a constant and $c$ is independent of $\phi$. This particular form of the matter Hamiltonian and the intial wave function were chosen to make it possible to find a solution. Nevertheless, we can write any possible matter dependence for this Hamiltonian as sum of solutions of the form (15). Using this form, we can rewrite the RHS of (13) as 


$$
-\frac{1}{3} \mu_{o}^{3} \gamma^{3} \kappa l_{p}^{2} \hat{H}_{m a t t} s_{\mu}=-\mu_{o}^{3} \gamma^{3} l_{p}^{3} \omega^{2} d_{j, l}(\mu) s_{\mu}
$$

where

$$
\omega^{2}=\frac{\tilde{\omega}^{2} \kappa \hbar^{2}}{6 l_{p}^{4}}
$$

We also introduced an exponential containing $\Gamma$ to eliminate the exponentials from the Hamiltonian constraint equation via the transformation $s \rightarrow s e^{i \mu \frac{\Gamma}{2}}$. The resulting equation can be written in iterative form

$$
\begin{array}{r}
s_{\mu+4 \mu_{o}}=\frac{l_{p}^{3}}{V_{\mu+5 \mu_{o}}-V_{\mu+3 \mu_{o}}}\left[\left(2-\gamma^{2} 4 \mu_{o}^{2}\left(\Gamma^{2}-\Gamma\right)\right)\left(\frac{V_{\mu+\mu_{o}}-V_{\mu-\mu_{o}}}{l_{p}^{3}}\right) s_{\mu}-\gamma^{3} \mu_{o}^{3} \operatorname{sgn}(\mu) \omega^{2} l_{p}^{3} d_{j, l}(\mu) s_{\mu}\right. \\
\left.-\left(\frac{V_{\mu-3 \mu_{o}}-V_{\mu-5 \mu_{o}}}{l_{p}^{3}}\right) s_{\mu-4 \mu_{o}}\right]
\end{array}
$$

which holds for all $\mu \neq-4 \mu_{o}$. None of these equations depend on $s_{0}$ which drops out of the equation entirely. This is why we have no equation for $\mu=-4 \mu_{o}$. Nevertheless, one can evolve though the classical singularity, $\mu=0$, since choosing $\mu=0$ in (18) is simply $s_{4 \mu_{o}}=s_{-4 \mu_{o}}$. In solutions where $\mu \neq 4 \mu_{o}$, (18) is always valid; thus, these are well defined around the zero volume state. The $\operatorname{sgn}(\mu)$ term is introduced because one has the freedom to choose the sign on the Hamiltonian constraint. The $\operatorname{sgn}(\mu)$ was chosen since there is no reason to expect different solutions in the classically disjoint regions $a<0$ and $a>0$.

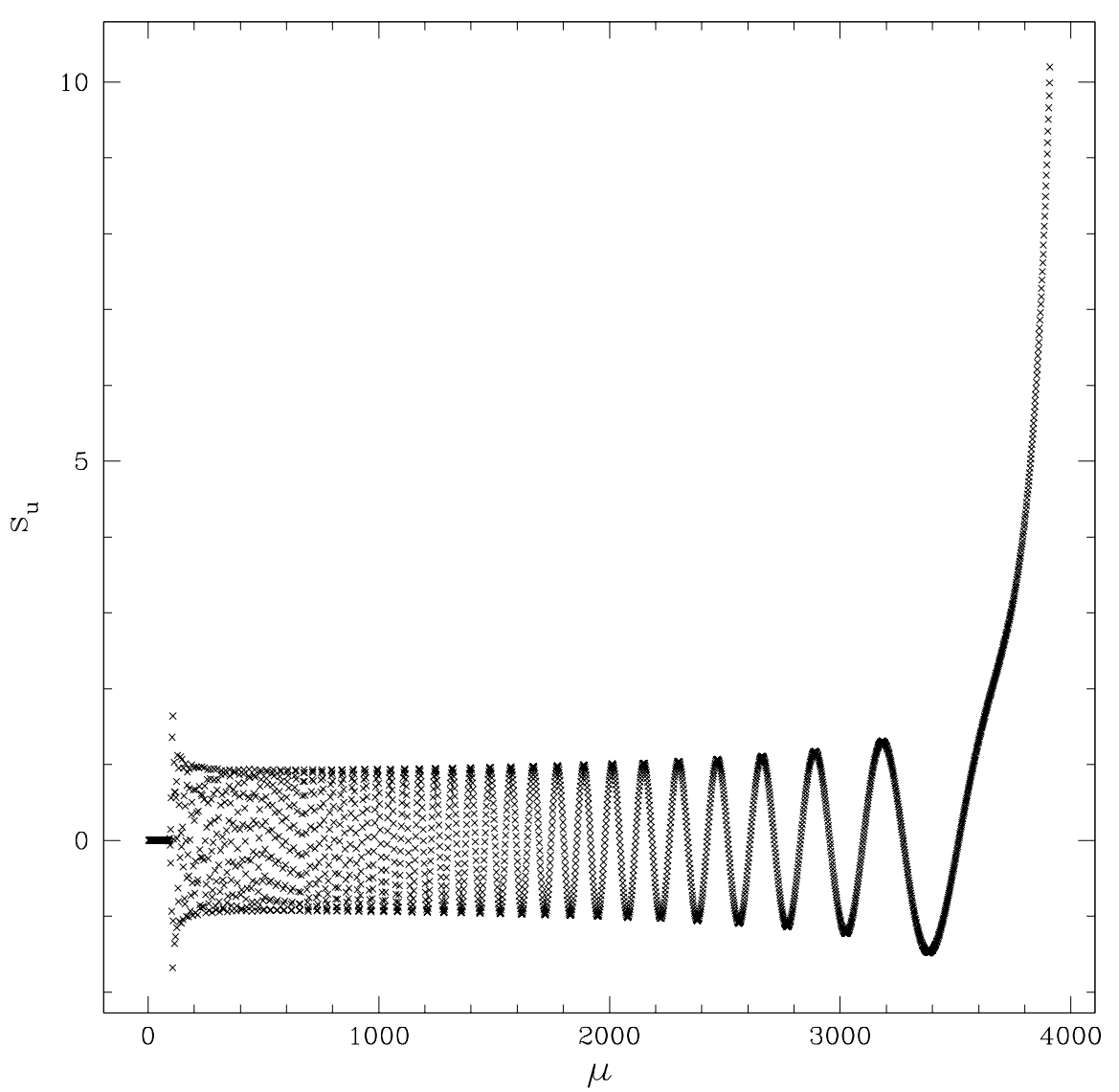

FIG. 1. This is the exact solution to the closed $\Gamma=\frac{1}{2}$ with $\omega=55$ and $j=20$. The initial value was set a $\mu=4 \mu_{o}$ with $\mu_{o}=\sqrt{3} / 4$. The state clearly diverges as we go to large scales. Since large $\mu$ is classically disallowed, we would like not to admit these states as physical possibilities. The factor $\exp (i \mu \Gamma / 2)$ has been left out of the solution. 
One can solve this equation iteratively by choosing 'initial' conditions at any particular point. In this model, we have 2 degrees of freedom, as long as the solution satisfies the equation at $\mu=4 \mu_{o}$. However, the form of the solutions shows the first problem with this approach. We have factored out the $e^{i \mu \frac{\Gamma}{2}}$ which will be present in all solutions. This factor implies that the state changes rapidly on scales near the plank scale. These oscillations on small scales also appear in the flat model and remain to be understood. However, in the flat model, one avoids such solutions on the grounds that they do not produce semi-classical physics. In this case, since the terms appear explicitly in the equation, we can still approximate (13) with a differential equation on large scales. Therefore, this is only a problem in the sense that it is not clear what this phase represents and how it relates to quantities that can be 'measured'.

Before we get to the properties of the exact solutions, we will look at the approximate behaviour on large scales. This will give a much better qualitative understanding of the solution than can be seen from the difference equation itself.

First of all, let us define the new variable $t_{\mu}$ such that

$$
t_{\mu}=e^{i \mu \frac{\Gamma}{2}}\left(V_{\mu+\mu_{o}}-V_{\mu-\mu_{o}}\right) s_{\mu}
$$

This satisfies the simpler equation

$$
t_{\mu+4 \mu_{o}}-2 t_{\mu}+t_{\mu-4 \mu_{o}}=-\omega^{2} \mu_{o}^{3} l_{p}^{6} d_{j, l}(\mu) \frac{t_{\mu}}{\left(V_{\mu+\mu_{o}}-V_{\mu-\mu_{o}}\right)}-\gamma^{2} 4\left(\Gamma^{2}-\Gamma\right) \mu_{o}^{2} t_{\mu} .
$$

When $\mu$ is large, the LHS of (20) can be approximated by a differential operator,

$$
t_{\mu+4 \mu_{o}}-2 t_{\mu}+t_{\mu-4 \mu_{o}}=16 \mu_{o}^{2} \frac{d^{2} t}{d \mu^{2}}=\frac{4}{9}\left(l_{p}^{2} \gamma\right)^{2} \mu_{o}^{2} \frac{d^{2} t}{d p^{2}}
$$

In the same limit, we have $d_{j, l}(\mu)=p^{-3 / 2}$. The final approximation for the difference in the volume eigenvalues. We can rewrite these in terms of $p$ using,

$$
V_{\mu+\mu_{o}}-V_{\mu-\mu_{o}}=\left(\frac{|\mu| \gamma l_{p}^{2}}{6}\right)^{3 / 2}\left(\left(1+\frac{\mu_{o}}{\mu}\right)^{3 / 2}-\left(1-\frac{\mu_{o}}{\mu}\right)^{3 / 2}\right) \approx\left(\frac{\gamma l_{p}^{2}}{6}\right)^{3 / 2} 3 \sqrt{\mu} \mu_{o}
$$

and $\sqrt{\mu}=\sqrt{\frac{6 p}{\gamma l_{p}^{2}}}$. From here we get

$$
V_{\mu+\mu_{o}}-V_{\mu-\mu_{o}}=\frac{\sqrt{p} \gamma l_{p}^{2}}{2}
$$

The end result is the Wheeler-DeWitt-like equation,

$$
p^{2} \frac{2}{9 \mu_{o}} \frac{d^{2} t}{d p^{2}}+p^{2} 2\left(\Gamma^{2}-\Gamma\right) \frac{t}{\mu_{o} l_{p}^{4}}+\omega^{2} t=0
$$

In the flat case $(\Gamma=0)$, one can find a solution by assuming $t=A p^{r}$. The equation gives the solution

$$
r=\frac{1}{2} \pm \sqrt{\frac{1}{4}-\frac{9 \omega^{2}}{2}}
$$

From this solution, one sees that there is a critical value of $\omega, \omega_{c}^{2}=\frac{1}{18}$ above which we get oscillatory solutions. Assuming $\omega>\omega_{c}$, the solution takes the form

$$
t=\sqrt{p}(B \exp (+i R \log (p))+C \exp (-i R \log (p)))
$$

where $R$ is the imaginary part of $r$. Recall that we absorbed volume terms into $t$. From $(23)$, we find that $s \propto \frac{t}{\sqrt{p}}$. Thus, in the large-scale limit, $s$ is purely oscillatory. Similar analysis has been performed for the flat model using quantizations of the Euclidian [16] and the Lorentzian [13] constraints and yield the same critical value.

In the closed case $\left(\Gamma=\frac{1}{2}\right)$, one can drop the matter term for large $p$ since it drops off as $\frac{1}{p^{2}}$ relative to the other terms. The resulting equation has simple solutions in terms of exponentials,

$$
t(\mu)=B \exp \left(-\frac{1}{4} \gamma \mu\right)+C \exp \left(+\frac{1}{4} \gamma \mu\right)
$$


where $B$ and $C$ are constants. The real exponentials are not surprising, given that large values of $a$ are classically disallowed. Like any conventional quantum mechanics problem, one would like to impose the boundary condition that $t \rightarrow 0$ as $|\mu| \rightarrow \infty$. Clearly one would set $C=0$ for large positive $\mu$, and $B=0$ for negative $\mu$. However, the iterative equation defines solutions for both positive and negative $\mu$.

Let us consider a solution where we pick some initial value $s_{4 \mu_{o}}=D e^{i \tilde{\omega} \phi}$. From (18), we find that

$$
s_{8 \mu_{o}}=\frac{l_{p}^{3}}{V_{9 \mu_{o}}-V_{7 \mu_{o}}}\left[\left(2+\gamma^{2} \mu_{o}^{2}\right)\left(\frac{V_{5 \mu_{o}}-V_{3 \mu_{o}}}{l_{p}^{3}}\right)-\gamma^{3} \mu_{o}^{3} \operatorname{sgn}(\mu) \omega^{3} l_{p}^{3} d_{j, l}(\mu)\right] s_{4 \mu_{o}} .
$$

Since $s_{8 \mu_{o}} \propto D, D$ will scale all values of the solution for positive $\mu$. Furthermore, when we set $\mu=0$, the constraint equation turns into $s_{4 \mu_{o}}=s_{-4 \mu_{o}}$. The evolution is symmetric in $\mu$ so $D$ will be present for all negative $\mu$ as well. Therefore, the solution will not depend on how we choose our initial value. One such exact solution is shown in figure 1. As one can see, the solution diverges at large $\mu$. Since we can only set an overall normalization, the only solution we have (other than $|\Psi\rangle=0$ ) is unphysical.

In the case where we define our initial value at $\mu \neq n 4 \mu_{o}$, where $n$ is an integer, there is no additional constraint on the solution from the zero volume state. However, because we no longer have the zero volume to eliminate terms, we do not have symmetric evolution in positive and negative $\mu$. Therefore, if we eliminate the divergence in the negative domain, we will still have the problem in the positive domain and vice versa. Figure 2 shows the state of the universe when we impose that $s_{\mu} \rightarrow 0$ for negative $\mu$. Clearly, the problem remains. This is very similar to the problem of producing the pre-classical solution in the flat model with the Lorentzian constraint [13]. In that case, one can only produce smooth solutions in either $\mu<0$ or $\mu>0$, but not both simultaneously.

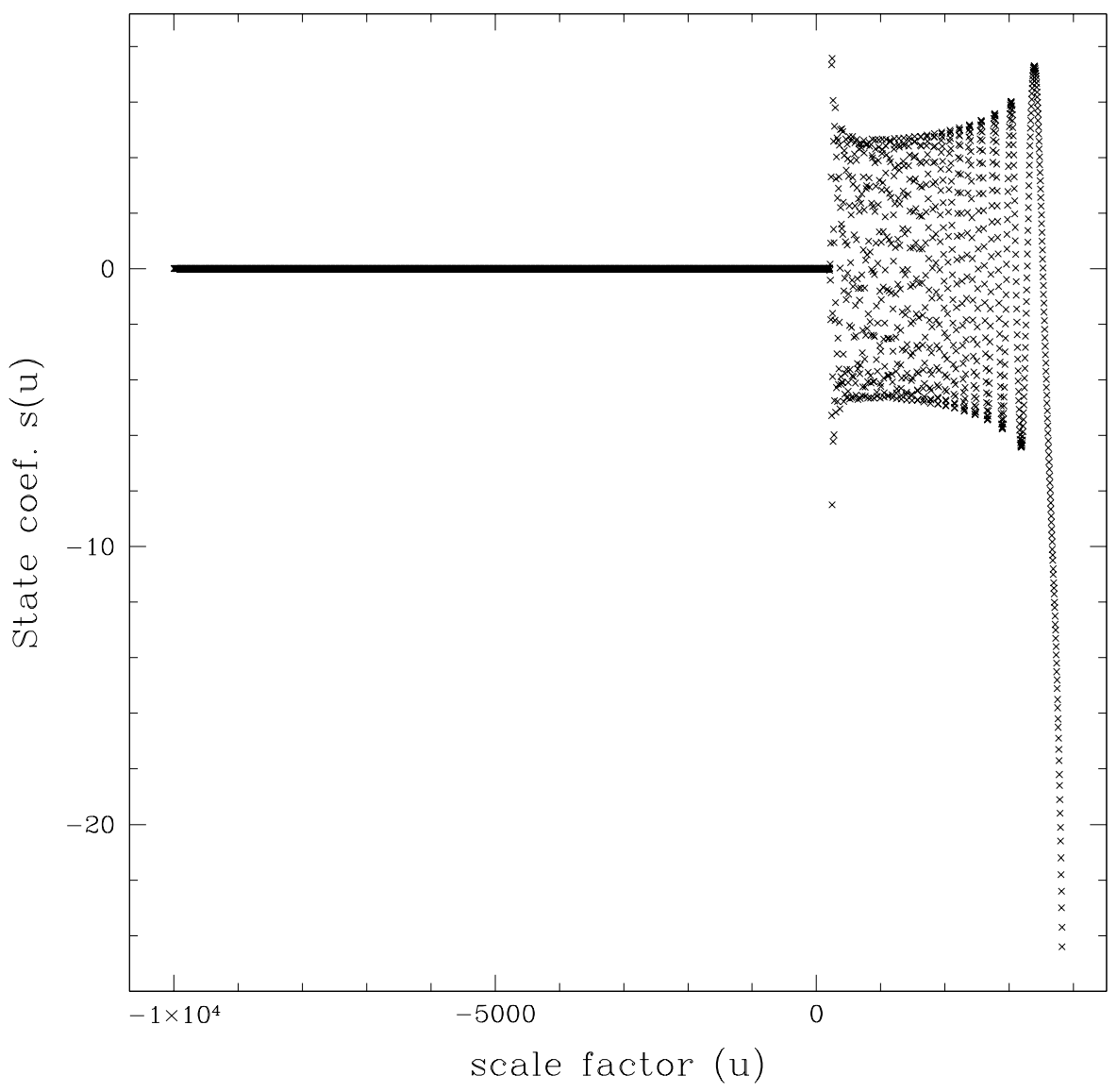

FIG. 2. This is the exact solution to the closed model $\Gamma=1 / 2$ with $\omega=55$ and $j=20$. The initial value was set a $\mu=3 \mu_{o}$ with $\mu_{o}=1$. The initial conditions have chosen to eliminate the growing solution in the $-\mu$ domain. However, it is clear we cannot eliminate the growing solutions in both positive and negative domains for the asymmetric model (i.e. the model where we don't hit zero volume). The factor $\exp (i \mu \Gamma / 2)$ has been left out of the solution. 
Solutions to this particular model have been presented [8] for the case where a cosmological constant is included. The solution avoids the difficulties presented here. In particular, one can rewrite the matter Hamiltonian as

$$
H_{\text {matt }}=a^{-3} p_{\phi}^{2}+a^{3} V_{o}
$$

where $V_{o}$ is the cosmological constant. The divergent solutions described above are not observed in this case because exponentially growing solutions are no longer a feature of the asymptotic solution. In particular, this introduces an additional term which dominates at large $\mu$. The resulting behaviour is described by

$$
\frac{d^{2} t}{d \mu^{2}}+|\mu| V t=0
$$

where $V=V_{o} \frac{1}{48} \mu_{o} \gamma^{3} \kappa l_{p}^{2}$. The solutions to (30) are defined in terms of Airy functions

$$
t=A^{\prime} A i\left(-V^{1 / 3}|\mu|\right)+B^{\prime} B i\left(-V^{1 / 3}|\mu|\right),
$$

where $A^{\prime}, B^{\prime}$ are constants. For $V_{o}>0$, the solutions are well behaved for all $\mu$. Coincidently (or perhaps not), this is the same condition that determines if large $a$ is classically disallowed. For a generic matter Hamiltonian and wave-function $\phi$ and $a$ will not decouple, but there will be regions of the $a-\phi$ plane that are classically disallowed, where we expect similar problems will arise.

There is reason to be concerned that the numerical results presented in figures 1 and 2 do not represent the exact solutions of the constraint equation. Because the solutions will take the form of exponentially growing and decaying solutions, numerical round-off errors may grow exponentially. This problem was tested explicitly. First of all, the solutions calculated using between 16 and 1000 digits all behaved in a similar manner and were within a few percent of each other where the divergences become large. Secondly, one can produce solutions that are decaying where solutions usually diverge by avoiding the constraint at zero (or leaving the opposite $\mu$ domain to diverge). These solutions are qualitatively different and near $\mu=0$ the ratio $s_{8 \mu_{o}} / s_{4 \mu_{o}}$ differ by orders of magnitude. This behaviour has been tested over a reasonable range in $\gamma, \mu_{o}, l, j$ and $\omega$ with similar behaviour in all cases.

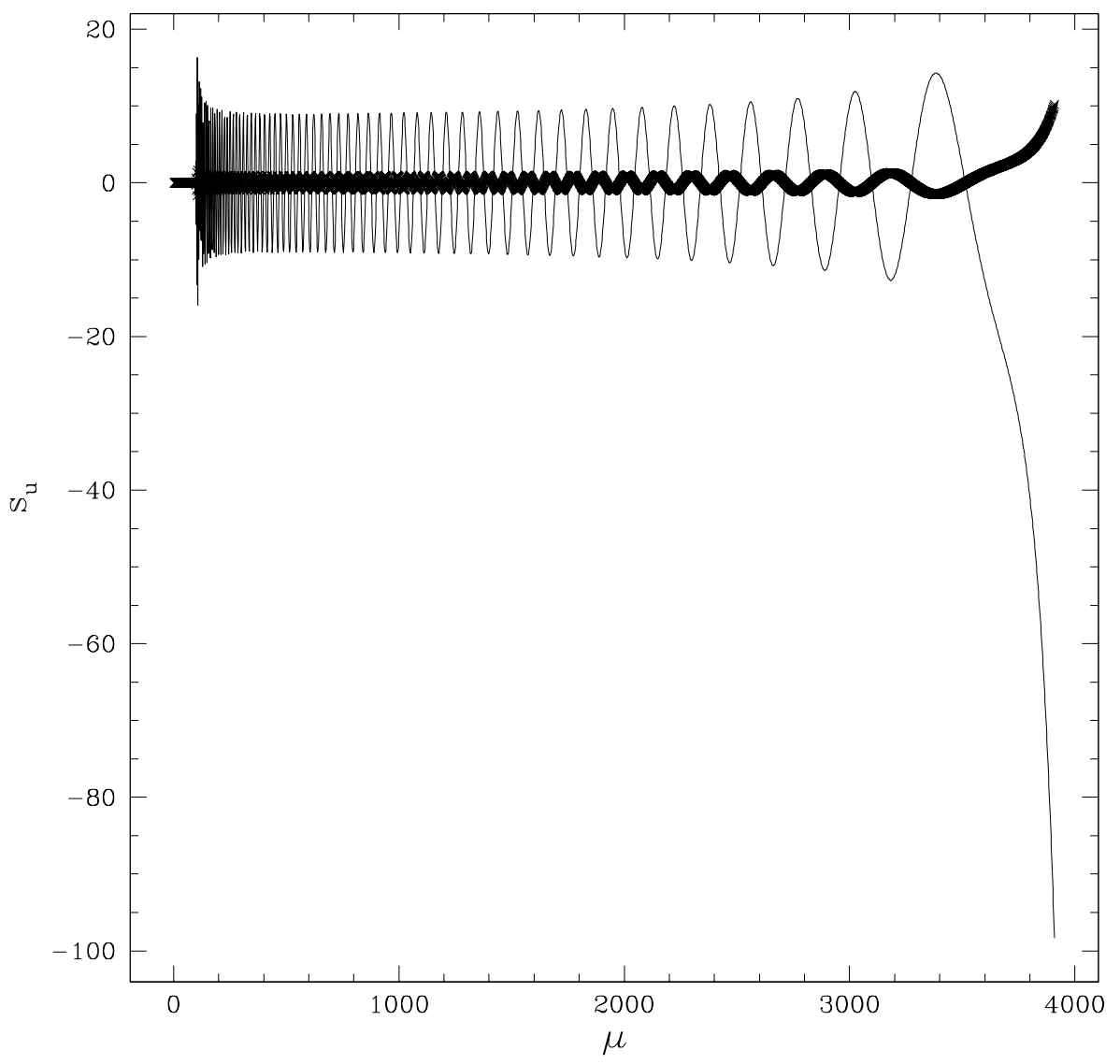


FIG. 3. This is the exact solution to the closed model $\Gamma=1 / 2$ with $\omega=55, j=20$ and $\gamma=\frac{\log 2}{\sqrt{3} \pi}$. The crosses represent the solution for $l=.45$ and solid line is for $l=.4475$. The initial value is set at $\mu=4 \mu_{o}$ and is equal in both cases. The change in the sign of the growing exponential part of the solution suggests that we can fix $l$ such that only the decaying solution remains.

Although these ambiguity parameters provide additional freedom in our solutions, it is unclear whether they can cure our problems or whether they simply relocate them. First of all, there have been arguments presented that should fix our choice of both $\gamma$ and $\mu_{o}$ [7]. Assuming these to be fixed, the only remaining parameters are $j$ and $l$. If we fix all values and adjust $l$ over the allowed range $(0,1)$, there are changes in the sign of the diverging exponential for fixed $s_{4 \mu_{o}}$. This suggests that we can fine tune $l$ such that the exponentially growing solution vanishes. Unfortunately, this fine-tuned value depends on $\omega$.

In figure 3, we have an example of the sign flip that occurs for a very small change in $l$ when $\omega=55$. However, if $\omega=50$, the solutions for the same two values of $l$ are virtually indistinguishable, as seen in figure 4 . Further choices of $l$ seem to suggest that there is no common $l$ in that range for both $\omega=50$ and $\omega=55$ that eliminates the unbounded solution. The same holds for a wide range of choices of $\omega \leq 500, j \leq 1000$ and $\gamma \leq 1$. Since the inverse density operator is defined on the kinematic Hilbert space, its definition should not depend on the form of the matter Hamiltonian or the particular state of the matter. In this particular model, one should be able to expand the solution as a sum over all $\omega$. One should not have to define $l(\omega)$, before doing so. Therefore, it seems unlikely that a choice of these parameters produce an acceptable model.

The bounded solutions achieved by fine-tuning $l$ have the feature of being sharply peaked around $\mu=0$, as suggested by figure 5 . This is an additional difficulty of the fine-tuned solutions. Not only does one need to fix $l(\omega)$, but one also needs to explain to meaning of the solutions. In particular, one should address why the only states are dominated by the smallest volume eigenstates.

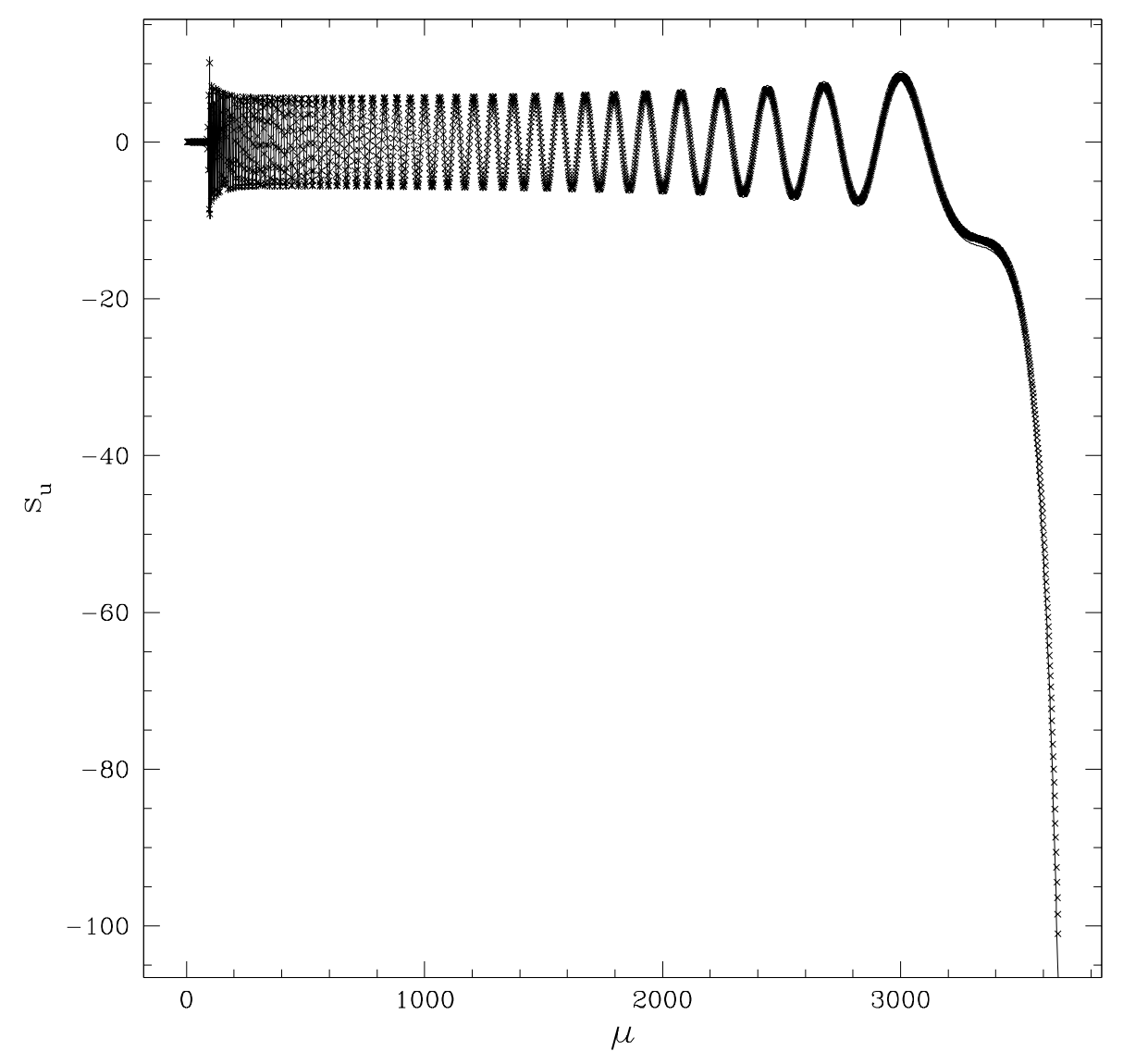


FIG. 4. This is the exact solution to the closed model $\Gamma=1 / 2$ with $\omega=50, j=20$ and $\gamma=\frac{\log 2}{\sqrt{3} \pi}$. The crosses represent the solution for $l=.45$ and solid line is for $l=.4475$. The initial value is set at $\mu=4 \mu_{o}$ and is equal in both cases. These solutions are nearly identical for all $\mu$. This suggests that there is no single value of $l$ that will produce bounded solutions for all choices of $\omega$.

Beyond fixing $l$, we could also fix other parameters in hopes of producing bounded solutions. For example, one could adjust the initial point (instead of assuming the symmetric solution) to some critical $\mu \in x 4 \mu_{o}$ where $x \in(0,1)$. This would eliminate the continuous spectrum of solutions for fixed $\omega$, etc in the same way we fixed $l$ or any of the ambiguity parameters. However, any one of these choices would depend on all the other parameters in the theory. Therefore, we can restrict one variable in order to produce bounded solutions but at the cost of introducing additional matter and ambiguity dependences into the states and the constraint. Therefore, one needs to ask whether it is sensible to have such a theory.

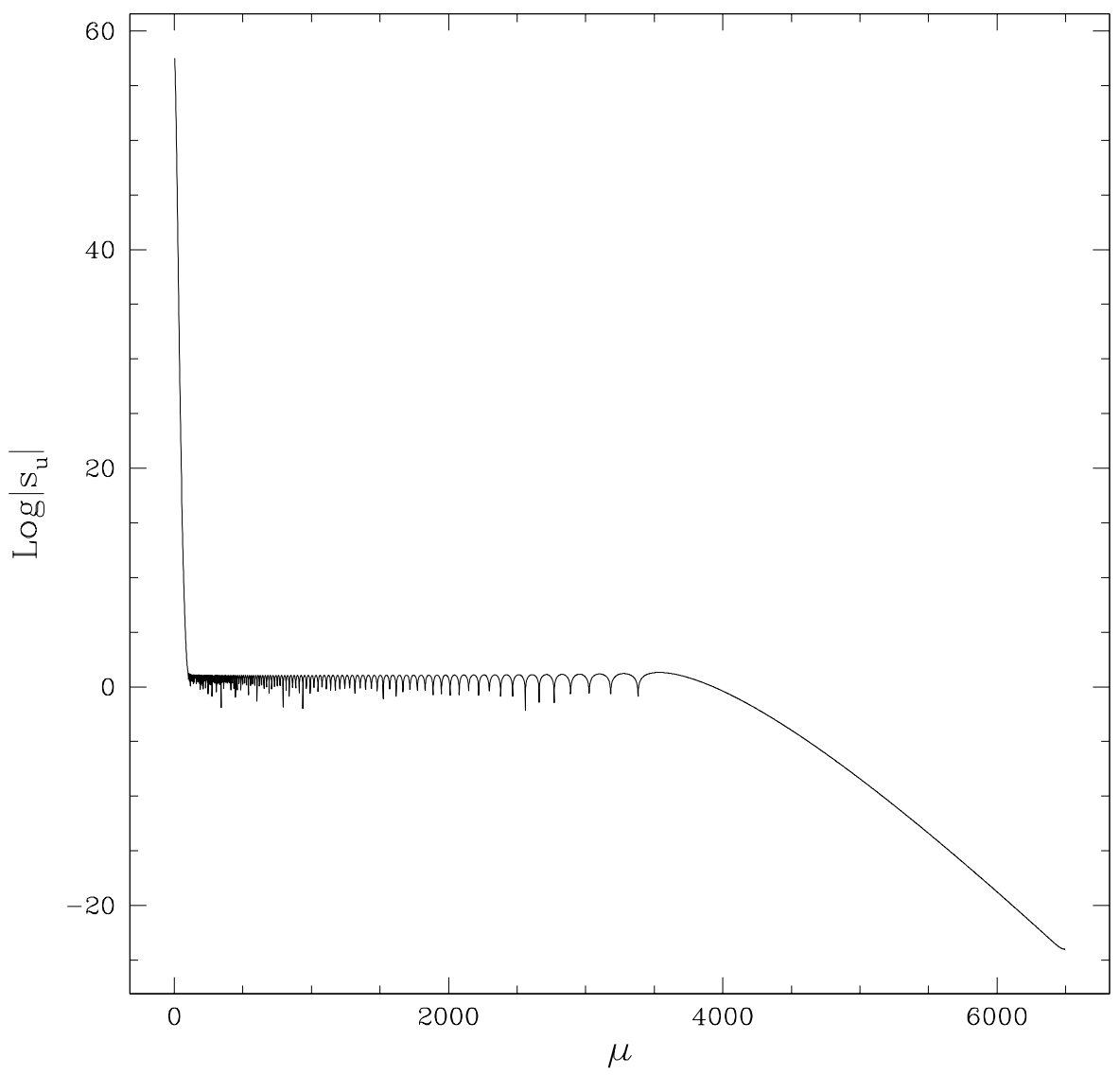

FIG. 5. This is a plot of the $\log$ of modulus of $s_{\mu}$ for the closed model $\Gamma=1 / 2$ with $\omega=55, j=20$ and $\gamma=\frac{\log 2}{\sqrt{3} \pi}$. The crosses represent the critical solution for $l=.447 \ldots$ in which the solution is bounded at large scales. The state is normalized such that the amplitude of the oscilation is of the same order as the other solutions. Thus, the value at $\mu=4 \mu_{o}$ is nearly $10^{60}$ compared to $10^{-50}$ in the other solutions shown. Clearly, the behaviour near $\mu=0$ dominates the solution.

\section{DISCUSSION AND CONCLUSION}

In the analysis of the behaviour of a massless scalar field in the closed model from ILQC, it seems that divergent behaviour of the solutions at large scales is unavoidable. In this analysis, we consider the solutions for a wide range of parameters (e.g. $j, l$ ). In every case, the solution oscillates at small scales but grows exponentially for large $\mu$. Attempts to constrain the ambiguity parameters through the boundary conditions seems to require significant matter dependence, and thus is an undesirable solution, if it works at all. 
When trying to produce a closed model using techniques from the full theory, one gets an unstable discrete equation. If the symmetry is used to make the constraint simpler to quantize, we get unbounded coefficients for the volume states in classically disallowed regions. If we are to interpret these solutions as representing a probability, then clearly we cannot allow the latter quantization. This assumption is not completely trivial. The physical meaning of these states is still unknown. Without an inner product and observables, the interpretation of the solution is not entirely clear (one possible interpretation can be found in [17]). Nevertheless, it seems unlikely that one can find an interpretation where the large-scale divergences are appropriate.

We are left with the problem of how to quantize the closed model in loop quantum gravity. From this point, it seems unlikely that a minor modification to the techniques applied to the flat model will be successful. Therefore, one needs to reconsider the techniques used from the first step in this procedure. One of the problems is that the mini-superspace treatment has removed the coordinate freedom one usually uses to eliminate the spin connection. If this is indeed the case, there must be a way to construct the cosmology from the full theory that does not suffer from this problem. Hence, the full theory might avoid these difficulties. This would also suggest that this mini-superspace model has less in common with the full theory than was intended.

Perhaps the most interesting aspect of the divergences that occur in this model is that they seem to occur under the same conditions that lead to a re-collapsing universe. The re-collapsing universe has typically been difficult to interpret in quantum cosmology. In this framework, it would be very difficult to understand the 'evolution' of a re-collapsing universe in terms of the volume states. It is interesting that our quantization seems to fail in the same place our framework would lead to significant interpretation problems.

It remains to be seen whether these types of problems rise in the Bianchi IX model from which this isotropic quantization was derived [15]. The homogenous solutions contain more degrees of freedom but will also have additional constraints and boundary conditions. One might hope that the problems found here result from limiting the phase space to classically isotropic solutions. If the homogenous solutions avoid these divergences then it would suggest that we might not need to worry about the difficulties in the isotropic case.

\section{ACKNOWLEDGEMENTS}

The DG would like to thank Bojan Losic for his comments and suggestions over the course of this work. WGU thanks the CIAR for support and both thank NSERC for their support of this work.

[1] M. Bojowald, Phys. Rev. Lett. 86, 5227 (2001)

[2] M. Bojowald, Phys. Rev. Lett 87, 121301 (2001)

[3] M. Bojowald, Phys. Rev. Lett 89, 261301 (2002)

[4] M. Bojowald and G. Date, Phys. Rev. Lett 92, 071302 (2004)

[5] T. Thiemann, Class.Quant.Grav. 15, 839 (1998)

[6] T. Thiemann, Class.Quant.Grav. 15, 1281 (1998)

[7] A. Ashtekar, M. Bojowald and J. Lewandowski, Adv.Theor.Math.Phys. 7, 233 (2003)

[8] M. Bojowald and K. Vandersloot, Phys. Rev. D67, 124023 (2003)

[9] M. Bojowald, Class.Quant.Grav. 19, 2717 (2002)

[10] M. Bojowald and G. Date, Class.Quant.Grav. 21, 121 (2004)

[11] M. Bojowald, gr-qc/0402053

[12] M. Bojowald, Class.Quant.Grav. 19, 5113 (2002)

[13] F. Hinterleitner, and S. Major, Phys. Rev. D 68, 124023 (2003)

[14] M. Bojowald, Class.Quant.Grav. 20, 2595 (2003)

[15] M. Bojowald, G. Date and K. Vandersloot, Class.Quant.Grav. 21, 1253 (2004)

[16] M. Bojowald and F. Hinterleitner, Phys.Rev. D66, 104003 (2002)

[17] D Colosi and C Rovelli, Phys.Rev. D68 104008 (2003) 